\title{
Spruce galactoglucomannan-stabilized emulsions as essential fatty acid delivery systems for functionalized drinkable yogurt and oat-based beverage
}

\author{
Fabio Valoppi ${ }^{1,2} \cdot$ Ndegwa Maina $^{1}\left(\right.$ Marja Allén $^{1} \cdot$ Roberta Miglioli $^{1,3} \cdot$ Petri O. Kilpeläinen $^{4} \cdot$ \\ Kirsi S. Mikkonen ${ }^{1,2}$ (iD
}

Received: 18 November 2018 / Revised: 14 March 2019 / Accepted: 24 March 2019 / Published online: 6 April 2019

(C) The Author(s) 2019

\begin{abstract}
The food industry has a considerable demand for functional foods, such as emulsions as delivery system for omega-6 and omega-3 fatty acids. Such delivery systems must be stabilized, ideally with a compound that fulfills the criteria for both functionality and sustainability. Spruce galactoglucomannans (GGM) are novel, wood-derived, natural, value-added, versatile, multi-purpose emulsifiers that can physically stabilize oil-in-water emulsions while simultaneously protecting the oil phase against oxidation. In this study, we present for the first time the use of GGM-stabilized emulsions as complex multicomponent delivery systems for omega-6 and omega-3 fatty acids, i.e., (1) cod liver oil in drinkable yogurt and (2) oat oil in a gluten-free vegan beverage. The emulsions and the resulting functionalized beverages were characterized in terms of their physical stabilities. In addition, functionalized, drinkable yogurt was characterized in terms of the oxidative stability of the oil. Results highlighted that oil droplets stabilized with GGM were stable during storage, against thermal treatment, upon addition into the beverages, and GGM protected cod liver oil against oxidation in drinkable yogurt. The results reported here highlight the vast potential for the use of wood lignocellulose-derived, multi-functional hydrocolloids in modern foods.
\end{abstract}

Keywords Galactoglucomannan · Emulsion · Drinkable yogurt · Oat-based beverage · Functional food

\section{Abbreviations \\ GGM Galactoglucomannan \\ HFY High-fat yogurt \\ LFY Low-fat yogurt}

Electronic supplementary material The online version of this article (https://doi.org/10.1007/s00217-019-03273-5) contains supplementary material, which is available to authorized users.

Kirsi S. Mikkonen

kirsi.s.mikkonen@helsinki.fi

1 Department of Food and Nutrition, University of Helsinki, Agnes Sjöbergin Katu 2, P.O. Box 66, FI-0014 Helsinki, Finland

2 Helsinki Institute of Sustainability Science, Faculty of Agriculture and Forestry, University of Helsinki, Helsinki, Finland

3 Department of Food, Environmental and Nutritional Sciences (DeFENS), Università degli Studi di Milano, Via G. Celoria 2, 20133 Milan, Italy

4 Natural Resource Institute Finland (LUKE), Tietotie 2, 02150 Espoo, Finland
OOE Oat oil emulsion

OBB Oat-based beverage

\section{Introduction}

Functional foods are a class of foodstuffs that has proven health benefits when consumed on a regular basis. They reduce the risk of specific chronic diseases and improve consumers' physical or mental well-being $[1,2]$. This class of foods has been recognized as one of the most innovative areas of research in the food industry [3]. Indeed, eating habits have a great effect on health, and consumers' increased awareness of this aspect, the increased cost of health care, and increased life expectancy have boosted the demand for functional foods considerably [2]. As a result, several food industries have launched new functional foods in the global market in the last decade. The global market for functional foods was valued $\$ 168$ billion in 2013 and is expected to be worth more than $\$ 300$ billion in 2020 [4].

Basically, functional foods are produced through the enrichment of foodstuff with bioactive and essential 
molecules, such as proteins, vitamins, carbohydrates (i.e., dietary fiber), and fatty acids (i.e., omega- 3 and omega- 6 fatty acids) [5] or adding pre- and pro-biotics [6, 7]. Beverages are consumed in large quantities throughout the world, and this has made them the perfect target for functionalization. Currently, the beverage sector is the most active sector in the production of functional foods, mainly due to their convenience and their providing the possibility of easily meeting consumers' needs. Commercial functional beverages include energy drinks, sport drinks, fruit and vegetable beverages, dairy-based beverages, and non-dairy-based beverages, such as oat-based beverages $[8,9]$. The extent and nature of the enrichment vary depending on the type of beverage. For example, sport drinks are enriched mainly with minerals and vitamins, whereas dairy-based functional beverages commonly are enriched with probiotics, minerals, fatty acids, and bioactive peptides [8].

The delivery of lipophilic compounds in a hydrophilic food matrix requires a delivery and protective system that can accommodate the added molecules, protect these molecules when they are added into the food matrix, and resist external stresses, i.e., technological processes, such as pasteurization, and the characteristics of the food matrix, such as low $\mathrm{pH}$ and high ionic strength [5]. To obtain delivery systems with tunable characteristics, a careful structural design approach must be used, and it must be coupled with technological approaches, such as emulsification $[5,10]$. Among the technologies that potentially could be used to produce a delivery system, emulsification has a great advantage because it can be used to generate extremely tunable systems with an encapsulation efficiency up to $100 \%$ [11]. However, the properties of the emulsifier are very important since emulsions serve as the delivery systems in functional foods. Indeed, emulsifiers must keep the emulsion stable when applied in the food matrix and under different environmental stresses [12]. As an additional point, the delivery of viable bioactive molecules that undergo oxidative degradation often requires the use of antioxidants to maintain their physiological effect in the final product [10].

Recently, we identified galactoglucomannan-rich (GGMrich) extracts obtained from industrial softwood by-products as novel, natural, value-added, emulsifying and stabilizing agents [13]. GGM are plant cell wall heteropolysaccharides, i.e., hemicelluloses, with similar carbohydrate structure as that of known hydrocolloids: guar gum galactomannan, locust bean gum galactomannan, and konjac glucomannan [14-16]. GGM consists of a linear backbone composed of $\beta$-(1 $\rightarrow 4)$-D-glucopyranosyl and partially acetylated $\beta$-( $1 \rightarrow 4)$-D-mannopyranosyl units, branched with $\alpha$ $(1 \rightarrow 6)$-D-galactopyranosyl side groups [17]. GGM also contain wood-derived extractives and phenolic co-travelers [18, 19], which may provide additional functionality for GGM [20]. GGM can be obtained in high yield from abundantly available wood sawdust using safe and environment-friendly pressurized hot water extraction (PHWE) [21]. Thus, they may be an interesting and sustainable option for emulsion stabilizers. We showed that GGM efficiently stabilized emulsions against coalescence [20, 22, 23] and protected the oil phase in emulsions against lipid oxidation [20, 24]. The presence of phenolic residues attached to GGMs was proposed to be the key element of the GGMs' interfacial and antioxidant properties. Indeed, it was hypothesized that the stabilizing mechanism involves phenolic compounds that deliver and anchor GGMs at the interface with oil droplets and induce steric stabilization [20].

GGM and lignin are classified as dietary fibers [25]. In addition to the stabilizing and antioxidant properties, GGM may also induce health-promoting effects, as reviewed by Pitkänen et al. [25]. After legislatively-required safety evaluations of novel foods [25], the characteristics of GGMs can make them a functionalizing and functional ingredient, which is very attractive in the food sector. GGM could be used as a multi-purpose ingredient to deliver and protect omega- 6 and omega- 3 fatty acids and obtain functional products. To develop new delivery/protective systems, they must be tested in complex multi-component food matrices.

In this study, we present two applications in which the functionality of GGM was exploited in drinkable yogurt (as an example of a dairy-based beverage) and oat-based beverages (as an example of a gluten-free, vegan beverage). The aim of this study was to explore the potential for the use of GGM-stabilized emulsions to deliver functional components in complex food matrices, i.e., to obtain functional foods. We focused on the physical stability of the functional foods as well as the ability of GGMs to protect sensitive compounds (such as labile fatty acids) against oxidation in a functional, drinkable yogurt. In particular, spruce GGMs were used to prepare emulsions that were rich in oils that contained omega- 6 and omega- 3 fatty acids, e.g., cod liver oil and oat oil. The results highlighted the vast potential for the use of wood lignocellulose-derived multifunctional hydrocolloids in modern functional foods.

\section{Materials and methods}

\section{Materials}

Oat oil containing rosemary extract as antioxidant (fatty acid composition in Table 1), $\beta$-glucan-rich flour containing $34-56 \% \beta$-glucan [26-28], and oat protein-rich powder containing 73-87\% proteins [26, 27], were kindly donated by Oy Karl Fazer Ab (Finland). 2.5 and $0.4 \%$ (w/w) dairy fat drinkable yogurts (Valio A + natural yogurt, Finland; fat content reported by the manufacturer) were purchased in a local market. Cod liver oil with $\alpha$-tocopheryl acetate and tocopheryl 
Table 1 Fatty acid composition (\% w/w) for oat oil and cod liver oil

\begin{tabular}{lll}
\hline Fatty acid & Oat oil & Cod liver oil \\
\hline C14:0 & 0.18 & 4.8 \\
C14:1 & n.d. & 0.12 \\
C16:0 & 15 & 11 \\
C16:1 & 0.19 & 10 \\
C18:0 & 1.7 & 2.1 \\
C18:1 (n-9) & 39 & 18 \\
C18:1 (n-7) & 0.73 & 5.2 \\
C18:2 (n-6) & 40 & 2.5 \\
C18:3 (n-3) & 1.6 & 1.1 \\
C20:0 & 0.79 & 14 \\
C20:1 (n-9) & 0.15 & n.d. \\
C20:2 (n-6) & n.d. & 0.34 \\
C20:3 (n-3) & n.d. & 0.14 \\
C20:4 (n-6) & n.d. & 0.34 \\
C20:5 (n-3) & n.d. & 9.6 \\
C22:0 & 0.01 & 6.3 \\
C22:1 & 0.18 & 0.74 \\
C24:0 & n.d. & 1.3 \\
C22:6(n-3) +C24:1 & n.d. & 12 \\
\hline
\end{tabular}

n.d. not detected

extract as antioxidants (Möller, Orkla Health, Norway) was purchased from a local market and the corresponding oil without the addition of antioxidant was kindly provided by Orkla Health (fatty acid composition in Table 1). Galactoglucomannans (GGMs) were extracted from spruce sawdust using a pressurized hot water flow-through extractor pilot plant [21]. The extract was then spray-dried and stored in pouches protected from light. Potassium sorbate was purchased from Fluka (Switzerland). Sodium hydroxide was purchased from J.T. Baker (The Netherlands). All solvents used for extractions were purchased from Sigma-Aldrich and Merck (Germany). Internal standard nonadecanoic acid methyl ester and GLC-68-D mixture of methyl esters of fatty acids were purchased from Nu-Check Prep, Inc. (USA).

\section{Sample preparation}

\section{Emulsions}

Concentrated emulsion containing cod liver oil A concentrated emulsion containing cod liver oil was prepared using $50 \%(\mathrm{w} / \mathrm{w})$ cod liver oil (with and without $\alpha$-tocopheryl acetate and tocopheryl extract), $40 \%$ (w/w) water, and $10 \%$ (w/w) GGMs. First, the GGMs were mixed with tap water at room temperature and left overnight to ensure maximum dissolution. Then, oil was added to the GGM solution, and an emulsion was obtained by homogenizing the system using a T-18 basic Ultra-Turrax high-speed homog- enizer (IKA, Germany) at 11,000 rpm for $4 \mathrm{~min}$ followed by $16,000 \mathrm{rpm}$ for $1 \mathrm{~min}$. The concentrated emulsion was stored at $4{ }^{\circ} \mathrm{C}$ and analyzed over a period of 29 days. The emulsion was prepared only using high-speed homogenization due to its high viscosity.

Oat oil emulsions We prepared four different types of oat oil-containing emulsions (OOE). To consider the dilution effect due to the preparation of an oat-based beverage (see below), first, concentrated emulsions were prepared and then diluted with tap water to the final ratios of GGMs and oil, i.e., $0.2 / 1.5,0.5 / 1,0.5 / 2$, and $0.8 / 1.5$. The emulsions were labelled as $\mathrm{OOE}_{0.2 / 1.5}, \mathrm{OOE}_{0.5 / 1}, \mathrm{OOE}_{0.5 / 2}, \mathrm{OOE}_{0.8 / 1.5}$, where the subscripts indicate the percentages of GGM and oil in the emulsion. The content of oil in OOE samples was selected to be in the same range of that of commercially available oat-based beverages. The GGMs were mixed with tap water at room temperature and left overnight to ensure their maximum dissolution. Then, oil was added, and the mixture was placed in an Ultra-Turrax homogenizer at 11,000 rpm for $5 \mathrm{~min}$ to obtain a coarse emulsion. Fine emulsions were obtained by homogenizing the coarse emulsions with three passes at a pressure of 800 bar using a Microfluidizer 110Y high-pressure homogenizer (Microfluidics, USA) configured with $75 \mu \mathrm{m}$ Y-type F20Y and $200 \mu \mathrm{m}$ Z-type H30Z chambers in series. Potassium sorbate was added to the emulsions at a $0.1 \%(\mathrm{w} / \mathrm{w})$ concentration to avoid microbial spoilage. The oat oil emulsions were stored at $22{ }^{\circ} \mathrm{C}$ and analyzed over a period of 28 days.

\section{Functionalized beverages}

Drinkable yogurt Functionalized drinkable yogurt was obtained by adding the concentrated cod liver oil emulsions (with and without added tocopheryl acetate and tocopheryl extract) to the yogurt that had 2.5 and $0.4 \%$ (w/w) dairy fat content, with final concentrations of cod liver oil of 0.5 and $1 \%(\mathrm{w} / \mathrm{w})$. The samples were labeled as follows: HFY0.5, HFY1, LFY0.5, and LFY1, where HFY and LFY mean high-fat yogurt $(2.5 \%)$ and low-fat yogurt $(0.4 \%)$, respectively. The number indicates the concentration of cod liver oil in the functionalized, drinkable yogurt. Concentrated emulsions also were prepared using cod liver oil without $\alpha$-tocopheryl acetate and tocopheryl extract to study the stability of the oil with respect to oxidation. The samples were mixed with an Ultra-Turrax homogenizer at 11,000 rpm for $1 \mathrm{~min}$ to facilitate the incorporation of the emulsion into the yogurt matrix and to ensure that the yogurt had the desired flow properties for drinking. Also, for comparison, drinkable yogurts without an added emulsion were treated using the same procedure. The samples were labeled as HFY0 and LFY0, and they were stored at $4{ }^{\circ} \mathrm{C}$ and analyzed over a period of 29 days. 
Oat-based beverage The oat-based beverages were obtained by adding both aqueous $\beta$-glucan-rich flour and oat protein suspensions to the oat oil emulsion. A $\beta$-glucanenriched suspension was obtained by stirring $3.3 \%(\mathrm{w} / \mathrm{v})$ $\beta$-glucan-rich flour in tap water overnight at room temperature (around $22{ }^{\circ} \mathrm{C}$ ). Then, the suspension was heated to $85^{\circ} \mathrm{C}$ and stirred for $2 \mathrm{~h}$ followed by stirring for an additional $2 \mathrm{~h}$ at room temperature. The oat protein suspension was obtained using a modified version of the method proposed by Yang et al. [29]. In particular, a suspension that contained $7.5 \%$ (w/v) of oat proteins was stirred overnight at room temperature. Then, the $\mathrm{pH}$ of the suspension was adjusted to 8 with $1 \mathrm{M} \mathrm{NaOH}$ to improve protein solubility, and the suspension was autoclaved at $115{ }^{\circ} \mathrm{C}$ for $12 \mathrm{~min}$. After reaching $80{ }^{\circ} \mathrm{C}$, the suspension was withdrawn from the autoclave and cooled to room temperature during magnetic stirring. Finally, the oat-based beverage was obtained by mixing the three components to obtain final $\beta$-glucan and protein concentrations of 1 and $3 \%(\mathrm{w} / \mathrm{w})$, respectively. The mixing was done by a T-18 basic Ultra-Turrax high-speed homogenizer (IKA, Germany) at 11,000 rpm for $2 \mathrm{~min}$. Four different samples were obtained, and they were labeled as $\mathrm{OBB}_{0.2 / 1.5}, \mathrm{OBB}_{0.5 / 1}, \mathrm{OBB}_{0.5 / 2}$, and $\mathrm{OBB}_{0.8 / 1.5}$, where the numbers in the subscripts refer to the percentage of GGMs and oil in the oat-based beverages, similar to those reported for the oat oil emulsions in paragraph 2.2.1.2. The content of oil in OBB samples was selected to be in the same range of that of commercially available oat-based beverages. Potassium sorbate was added to the samples at a $0.1 \%$ (w/w) concentration to avoid microbial spoilage. The final $\mathrm{pH}$ of OBB samples was 6.9. Selected samples were pasteurized at $80{ }^{\circ} \mathrm{C}$ for $10 \mathrm{~min}$. Samples were stored at room temperature (around $22{ }^{\circ} \mathrm{C}$ ) and analyzed over a period of 28 days.

\section{Analytical determinations}

\section{Fatty acid composition}

The composition of fatty acids of cod liver oil and oat oil was assessed by acid-catalyzed esterification and transesterification followed by gas chromatographic (GC) quantification. The esterification and transesterification were performed using $1 \%$ sulfuric acid in methanol as described by Christie and Han [30], with some modifications. Around $50 \mathrm{mg}$ of oils were first dissolved in toluene, added with $1 \%$ sulfuric acid in methanol and incubated at $85^{\circ} \mathrm{C}$ for $60 \mathrm{~min}$. Esterified fatty acids were extracted using heptane after addition of saturated sodium chloride solution. The heptane layer was collected and dried over anhydrous sodium sulfate. The GC analysis was performed using the GC-FID equipment and the method described by Yang et al. [31], with some modifications. Briefly, the fatty acid methyl esters were analyzed injecting in a split mode $(1: 15) 1 \mu \mathrm{L}$ of sample at $250{ }^{\circ} \mathrm{C}$ in a GC-FID (Agilent $6890 \mathrm{~N}$, USA) equipped with a fused silica capillary column Omegawax ${ }^{\text {TM }} 1250$ $\left(30 \mathrm{~m} \times 0.25 \mathrm{~mm} \times 0.25 \mu \mathrm{m}, \mathrm{SUPELCO}^{\circledR}\right.$, USA). Helium at $1.1 \mathrm{~mL} / \mathrm{min}$ was used as carrier gas. The oven temperature was programmed to increase from $180{ }^{\circ} \mathrm{C}$ with a $3{ }^{\circ} \mathrm{C} / \mathrm{min}$ to $220{ }^{\circ} \mathrm{C}$ and finally increased with a $6{ }^{\circ} \mathrm{C} / \mathrm{min}$ to $250^{\circ} \mathrm{C}$ (with a 12-min final hold). Methyl esters of fatty acids were identified using a GLC-68-D (Nu-Chek Prep. Inc., USA) commercial mixture of methyl ester of fatty acids and quantified using nonadecanoic acid methyl ester as internal standard.

\section{Size and distribution of the oil droplets}

The Sauter $(D[3,2])$ and De Brouckere $(D[4,3])$ mean diameters and the droplet size distribution of emulsions were determined using a Mastersizer 3000 static light scattering apparatus mounted with a Hydro EV dispersion accessory (Malvern Instruments Ltd, UK). The instrument was controlled by the Mastersizer v.3.62 (Malvern Instruments Ltd, UK) application software. Before conducting the analyses, the emulsions were turned upside down gently ten times. Then, the emulsions were added directly into the dispersion accessory which allowed them to be diluted to avoid multiple scattering effects. The refractive indexes of water and oil were 1.33 and 1.47 , respectively. The mean diameters and size distribution of the oil droplets were calculated as an average of three measurements for each of the two samplings performed on each emulsion.

\section{Microscopy}

All emulsions, plain and functional yogurts, and oat-based beverages were analyzed using an AxioScope A1 optical microscope (Carl Zeiss Inc., Germany) connected to a Axiocam MRm digital camera (Carl Zeiss, Inc., Germany). Before analysis, the samples were mixed by turning the container upside down ten times. One drop of sample was placed in the middle of a glass slide and a glass cover slip was centered above the drop. The samples were analyzed using both $40 \times$ and $100 \times$ objective. Images were acquired using Axiovision v.4.7.1.0 (Carl Zeiss, Inc., Germany) application software.

\section{Flow curves}

Flow curves of functionalized drinkable yogurts, oat oil emulsions, and oat-based beverages were obtained using a Rheolab QC rheometer (Anton Paar GmbH, Germany) controlled by Rheoplus v.3.61 software (Anton Paar GmbH, Germany). The experiments were performed using a concentric cylinder geometry CC27 (cylinder diameter: $26.66 \mathrm{~mm}$, cup diameter: $28.84 \mathrm{~mm}$ ), and the measurements were conducted at $10^{\circ} \mathrm{C}$ for drinkable yogurt and at $22^{\circ} \mathrm{C}$ for oat oil-containing emulsions 
and the oat-based beverage. An RC6-RCS temperature controller unit (Lauda, Germany) was connected with the rheometer and used to control the temperature. Before the samples were analyzed, they were mixed by turning the container upside down ten times. Flow curves were obtained by increasing the shear rate step-wise from 2 to $200 \mathrm{~s}^{-1}$.

\section{Sedimentation}

The stability against the gravitational separation of oat-based beverages $\left(\mathrm{OBB}_{0.2 / 1.5}, \mathrm{OBB}_{0.5 / 1}, \mathrm{OBB}_{0.5 / 2}\right.$, and $\left.\mathrm{OBB}_{0.8 / 1.5}\right)$ was determined using the method proposed by Mirhosseini et al. [32]. In particular, $10 \mathrm{~mL}$ of the oat-based beverage was transferred into a 15-mL Falcon tube. During storage, the volume $(\mathrm{mL})$ of sediment was measured, and the stability index (SI) was computed as follows:

SI $(\%)=\frac{V_{\mathrm{T}}-V_{\mathrm{S}}}{V_{\mathrm{T}}} \times 100$

where $V_{\mathrm{T}}$ is the total volume $(\mathrm{mL})$ of the sample, $V_{\mathrm{S}}$ is the volume $(\mathrm{mL})$ of the syneresis layer in the top of the Falcon tube, and SI is the mean percentage \pm SE derived from the two experimental replicates.

\section{Secondary oxidation products}

The secondary oxidation products of plain and functionalized drinkable yogurts (HFY0, HFY0.5, HFY1, LFY0, LFY0.5, and LFY1) that contain cod liver oil without $\alpha$-tocopheryl acetate and tocopheryl extract were extracted by solid phase microextraction (SPME) and analyzed with gas chromatography coupled with mass spectrometry (GC-MS), using the same method described by Lehtonen et al. [24]. Three replicates consisting of approximately $2 \mathrm{~g}$ of sample that had been weighed in amber glass vials, hermetically sealed, and stored at $4{ }^{\circ} \mathrm{C}$ in the dark were withdrawn for analysis. Briefly, the volatile compounds were adsorbed onto a DVB/CAR/PDMS fiber (Supelco, USA), released in the GC-MS injector at $250^{\circ} \mathrm{C}$ for $10 \mathrm{~min}$, and analyzed using an HP 6890 series GC coupled with an Agilent 5973 MS (Agilent Technologies Inc., USA). They were separated using a SPB-624 capillary column (Supelco, USA) and a temperature program from 40 to $200{ }^{\circ} \mathrm{C}$ with helium at a flow rate of $0.7 \mathrm{~mL} / \mathrm{min}$ with the MS running in full-scan mode. The compounds were identified based on the comparison of their retention times and mass spectra with those of the standards.

\section{Data analysis}

All determinations were expressed as the mean \pm standard error (SE) of at least two measurements from two replicates $(n \geq 2 \times 2)$, if not otherwise specified. Linear regression
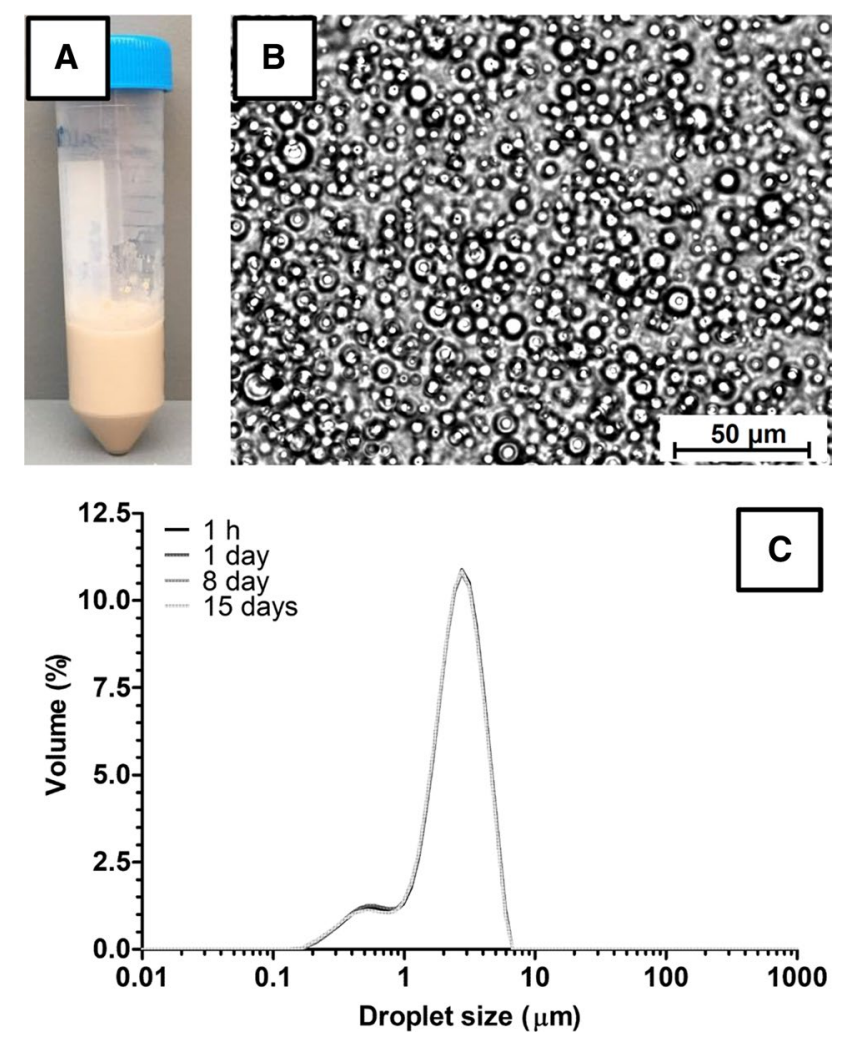

Fig. 1 a Macroscopic image of the freshly prepared, concentrated emulsion that contained 50\% cod liver oil and 10\% GGM, b its relevant optical microscopy image, and $\mathbf{c}$ droplet size distribution of emulsion during storage at $4{ }^{\circ} \mathrm{C}$

analysis by least-squares regression was performed, and the goodness of fit was evaluated on the basis of the statistical parameters of fitting $\left(R^{2}, p\right.$ value, and standard error) and the residual analysis. Statistical significance was considered for $p<0.05$.

\section{Results and discussion}

\section{Cod oil emulsions}

The first example of product functionalization was on drinkable yogurt using an emulsion stabilized by GGM highly loaded with omega-6 and omega-3 rich oil. Drinkable yogurt is a food product that is consumed worldwide, and, currently, it is used as a target food for functionalization [8]. We will first explore the highly oil-loaded emulsion, which was prepared to avoid the dilution effect when the emulsion was added to the yogurt.

Figure 1a shows the macroscopic appearance of GGMstabilized emulsion containing 50\% (w/w) cod liver oil (hereafter called concentrated emulsion). The sample appeared as a brownish dispersion. During the extraction 
process, lignin and other extractives were recovered along with GGM [21]. Lignin is a macromolecule that is composed of polymerized monolignols (phenolic compounds) [33], and its color ranges from light brown to dark brown [34]. The presence of lignin and other extractives probably is responsible for the brownish color of the concentrated emulsion.

From a microscopic perspective, the concentrated emulsions appeared as a polydisperse system in which a large number of oil droplets with different diameters was observed (Fig. 1b). After 15 days of storage at $4{ }^{\circ} \mathrm{C}$, no differences in the morphology of the oil droplets were observed (data not shown). Then, the stability of the concentrated emulsion over time was confirmed by the droplet size distribution (Fig. 1c). A main peak at around $3 \mu \mathrm{m}$ with a shoulder at a lower size was visible for all storage times. The concentrated emulsion was physically stable for 15 days of storage at $4{ }^{\circ} \mathrm{C}$, exhibiting a constant $D[3,2]$ and $D[4,3]$ of $1.75 \pm 0.05$ and $2.74 \pm 0.02$, respectively. The stability can be attributed to both GGMs' stabilizing ability and the high mass fraction of oil in the concentrated emulsion. Indeed, it is known that a high mass fraction of oil increases the viscosity of emulsions, which, in turn, retards the possible breakdown mechanisms, thereby increasing the stability of the emulsion [12]. Concentrated emulsions can be used as ingredients to add or dilute to obtain the final product. The fact that concentrated emulsions contain high amounts of oil allows these emulsions to be stored for prolonged periods of time and be added at the desired time. Also, since concentrated emulsions are ingredients, they reduce the costs related to the storage and transportation of the final product $[35,36]$.

\section{Drinkable yogurt}

The concentrated emulsion was added to drinkable yogurt with 2.5 or $0.4 \%(\mathrm{w} / \mathrm{w})$ fat content to obtain final concentrations of 0.5 and $1 \%(\mathrm{w} / \mathrm{w})$ of cod liver oil in the final product (samples HFY0.5, HFY1, LFY0.5, LFY1). The addition of the emulsion altered the color of the drinkable yogurts slightly, from white to slightly brown (Fig. 2). It is known that color changes in food products modify the perception and acceptance of the products [37]. However, consumers tend to accept sensorial changes in food products that are enriched or functionalized compared to the respective plain foods [38].

Figure 2 shows the micrographs of the plain (HFY0 and LFY0) and functionalized drinkable yogurts (HFY0.5, HFY1, LFY0.5, LFY1). Cod liver oil droplets in HFY0.5, HFY1, LFY0.5, LFY1 were distributed evenly in the samples and incorporated into the protein-yogurt matrix. No differences were noted among the samples, indicating that the presence of milk fat did not affect the stability of the oil droplets. Also, no morphological differences were
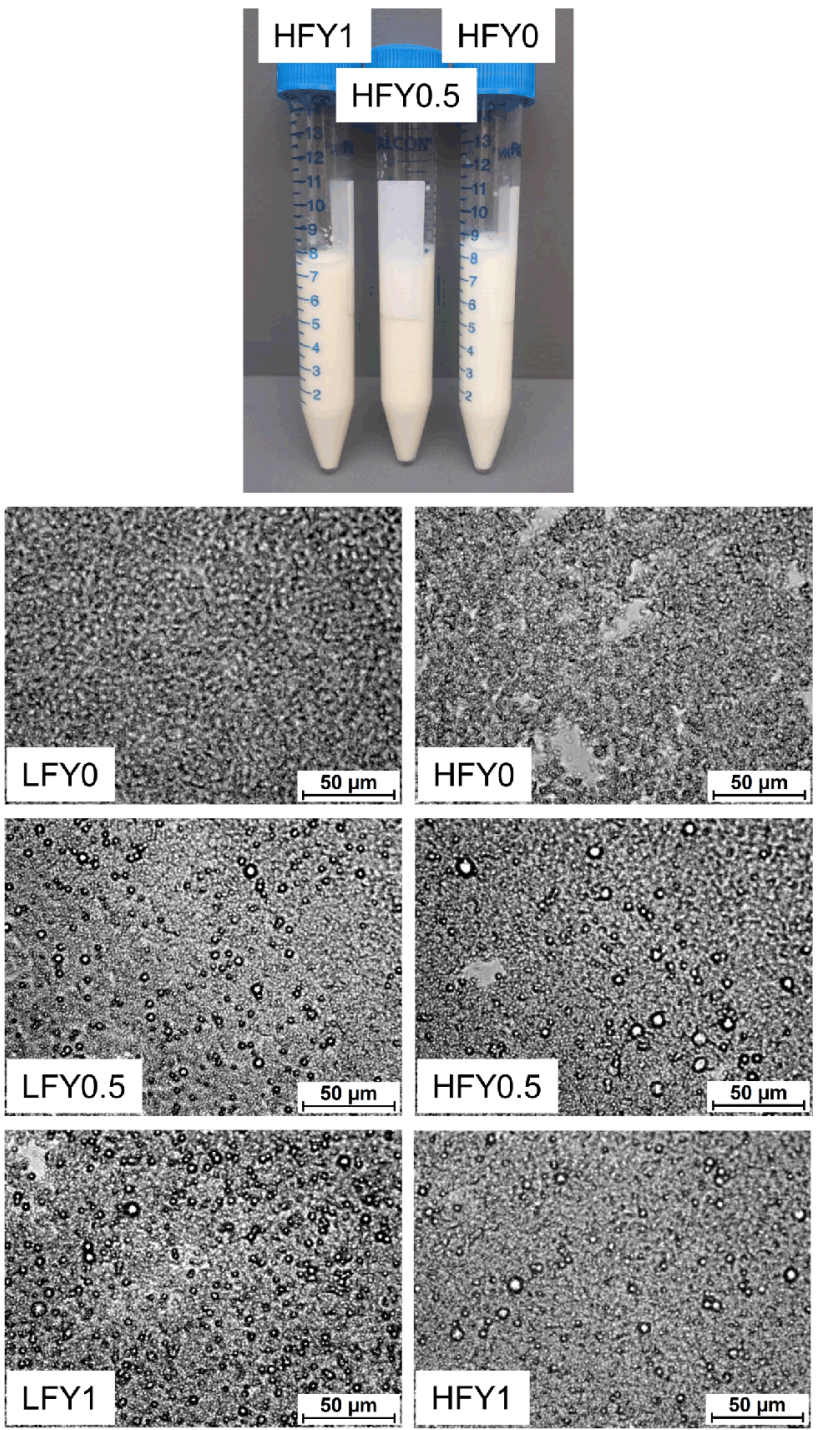

Fig. 2 Macroscopic images (left to right) of HFY1, HFY0.5, and HFY0 and optical microscopy images of LFY0, LFY0.5, LFY1, HFY0, HFY0.5, and HFY1

noted between the oil droplets in the concentrated emulsion and the oil droplets dispersed in the drinkable yogurts (cf. Figs. $1 b$ and 2). The stability of oil droplets in drinkable yogurt can be explained by the reduced/absent interactions between GGMs and caseins and the viscosity of the system. Yogurt is formed upon acidification induced by fermentation, and a three-dimensional network of clusters and chains of caseins is formed due to reduced electrostatic repulsion between the casein molecules [39]. The zeta potential of caseins is slightly negative (close to zero) at $\mathrm{pH} 3.5$, and it decreases as the $\mathrm{pH}$ value increases [40]. In acidic conditions $(\mathrm{pH}=4.5)$, GGMs have shown a zeta potential of about $-10 \mathrm{mV}$ [13]. Thus, when the concentrated emulsion comes in contact with the casein network of the yogurt, the weak electrostatic forces that act between the GGMs and the 
caseins permit the incorporation of the concentrated emulsion into the yogurt's protein matrix.

To understand the effect of the addition of the emulsion on the mechanical properties of the drinkable yogurts, the flow curves of HFY0, HFY1, LFY0, and LFY1 were determined (Fig. 3). Both yogurts (LFY0 and HFY0) had pseudoplastic behavior (Fig. 3). HFY0 had a flow curve that shifted to lower values than that of LFY0. This shift was due to the suppression effect that fat has on the elastic component of the yogurt's protein network [41]. However, even though HFY0 and LFY0 showed different flow behaviors (Fig. 3), it was possible to incorporate the concentrated emulsion in both types of yogurts. It is important to remember that viscosity can have a significant effect on the stability of the oil droplets in the yogurt's protein matrix. Even though a small amount of emulsion was present in HFY1 and LFY1, a slight modification of the enriched samples' flow curves was observed (Fig. 3). In particular, the curves were shifted at higher shear stresses, indicating an increase in the viscosity. The same shift was observed for HYF0.5 and LFY0.5, even though the magnitude of the shift was lower (data not shown). It is known that oil droplets are responsible for increases in viscosity due to the augmented friction among the particles, while oil volume fraction is responsible for the magnitude of the increase [12]. However, in this study the magnitude of the increase is negligible from a practical perspective due to people's low sensitivity in detecting small changes in viscosity [42]. No changes in the flow behavior were observed during 15 days of storage at $4{ }^{\circ} \mathrm{C}$ (cf. Figure 3 with Fig. S1 in Supporting Information).

The volatile oxidation products were evaluated over an extended storage period of 29 days at $4{ }^{\circ} \mathrm{C}$ to evaluate the protective effect of GGMs on the cod liver oil that was mixed into the drinkable yogurts. No secondary oxidation

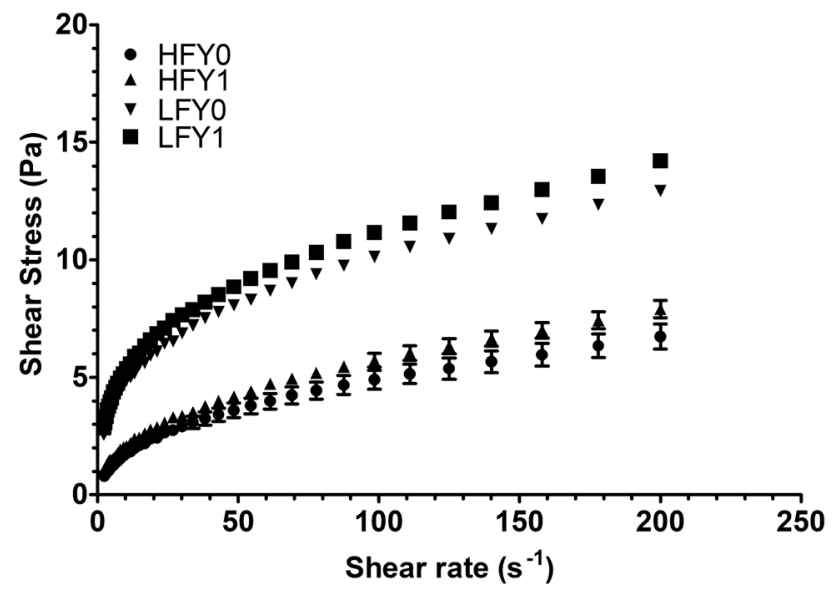

Fig. 3 Flow curves of freshly prepared samples HFY0, HFY1, LFY0, and LFY1: the analyses were conducted at $10{ }^{\circ} \mathrm{C}$. The bars represent standard error products were detected during storage in any of the functionalized yogurts (HFY0.5, HFY1, LFY0.5, and LFY1). Additionally, no differences among the samples were detected in the chromatograms. Thus, GGMs were able to protect cod liver oil against oxidation in a complex matrix, such as yogurt, as highlighted by the absence of secondary oxidation products, i.e., aldehydes, alcohols, and ketones, during storage. Secondary oxidation products potentially can be toxic to people since they are absorbed readily and transported to tissues where they can react with proteins, phospholipids, and nucleic acids [43]. The antioxidant property of GGM is attributed to the free and bound phenolic compounds that react with oxygen, thereby protecting unsaturated fatty acid moieties in triacylglycerol molecules [20, 24]. Thus, it is possible to use GGM to deliver the cod oil in yogurt that is rich in omega- 6 and omega- 3 fatty acids and avoid the formation of potentially toxic molecules and rancidity in drinkable yogurt during storage.

\section{Oat oil emulsions}

In this second case study, we report the use of emulsions containing oat oil and stabilized by GGM as a way of obtaining functional, oat-based beverages. Plant-based, non-dairy beverages are a rapidly growing segment of functional food products [44]. Oat-based beverages are an example of gluten-free milk alternatives for vegans. Oats are a gluten-free cereal that can be consumed safely by celiac patients, and this cereal possibly can contribute to improvements in the quality of their lives [45]. Oat $\beta$-glucan, contained in oat beverages, has a very high capacity for reducing cholesterol, postprandial glucose, and insulin; its capacity is even higher than that of barley $\beta$-glucan [46, 47]. Based on the beneficial effect of oat $\beta$-glucan, the oat-based beverage presented here was made from oat $\beta$-glucan-rich flour, oat proteins, and an emulsion stabilized using GGMs that contained oat oil, which is rich in omega- 6 fatty acids (Table 1).

All of the emulsions that contained oat oil were visually homogeneous with a viscosity comparable to that of water (around $1 \mathrm{mPa} \cdot \mathrm{s}$ at $22{ }^{\circ} \mathrm{C}$ ). During storage, a thin creaming layer was observed visually in all of the emulsions except $\mathrm{OOE}_{0.5 / 1}$. All of the emulsions, except $\mathrm{OOE}_{0.5 / 2}$, exhibited good stability over time. Samples with a higher GGM-tooil ratio (Fig. 4, $\mathrm{OOE}_{0.8 / 1.5}$ and $\mathrm{OOE}_{0.8 / 1.5}$ ) had a main peak around $0.1 \mu \mathrm{m}$, followed by minor peaks at higher droplet sizes. During storage, the main peak remained stable, while the minor peaks shifted slightly at higher values, indicating good stability against coalescence. Emulsions with lower GGM-to-oil ratios (Fig. 4, $\mathrm{OOE}_{0.2 / 1.5}$ and $\mathrm{OOE}_{0.5 / 2}$ ) had a main peak at $0.3 \mu \mathrm{m}$ and other peaks or shoulders at sizes greater than $2 \mu \mathrm{m}$. In both emulsions, the peak at the lower size remained stable for the entire storage period, while the peaks and shoulders that were larger moved to higher values, 
Fig. 4 Droplet size distribution for $\mathrm{OOE}_{0.8 / 1.5}, \mathrm{OOE}_{0.5 / 1}$, $\mathrm{OOE}_{0.2 / 1.5}, \mathrm{OOE}_{0.5 / 2}$ during storage at $22{ }^{\circ} \mathrm{C}$. Subscripts in abbreviations of the samples represent the percentages of GGM and oil
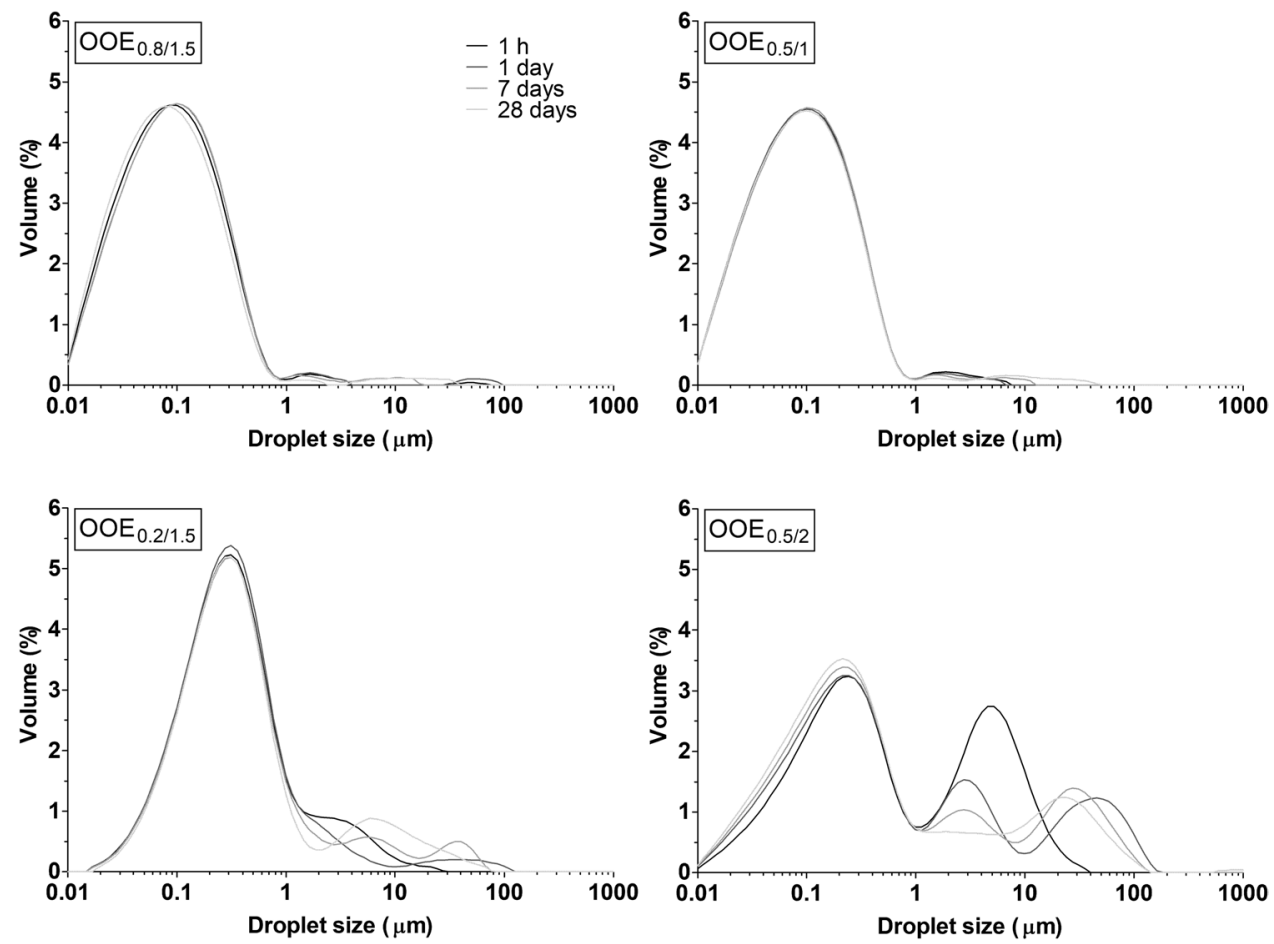

indicating a possible aggregation or coalescence of the oil droplets. This phenomenon was more evident in $\mathrm{OOE}_{0.5 / 2}$ than in $\mathrm{OOE}_{0.2 / 1.5}$. The stability of the emulsions followed the order: $\mathrm{OOE}_{0.8 / 1.5}=\mathrm{OOE}_{0.8 / 1.5}>\mathrm{OOE}_{0.2 / 1.5}>\mathrm{OOE}_{0.5 / 2}$, the latter sample being the most unstable one.

We also assessed the stability of the emulsion by evaluating the Sauter $(D[3,2])$ and De Brouckere $(D[4,3])$ mean diameters; $D[3,2]$ remained constant during storage for all of the emulsions, but $D[4,3]$ tended to increase with different magnitudes during storage. In particular, $D$ $[4,3]$ increased slightly for $\mathrm{OOE}_{0.8 / 1.5}$ and $\mathrm{OOE}_{0.5 / 1}$ (initial: $0.25 \pm 0.05$, final: $0.45 \pm 0.05 \mu \mathrm{m}$ ) and increased for $\mathrm{OOE}_{0.2 / 1.5}$ (initial: $0.88 \pm 0.02$, final: $2.21 \pm 0.10 \mu \mathrm{m}$ ), while a more marked increase was observed for $\mathrm{OOE}_{0.5 / 2}$ (initial: $2.76 \pm 0.01$, final: $9.39 \pm 0.10 \mu \mathrm{m}$ ).

The increase of the diameters of the oil droplets and the presence of flocculated oil droplets also were evaluated using optical microscopy. As an example, Fig. 5 (left) shows the freshly prepared emulsion of $\mathrm{OOE}_{0.8 / 1.5}[0.8 \%(\mathrm{w} / \mathrm{w})$ GGM and $1.5 \%(\mathrm{w} / \mathrm{w})$ oil] and after 28 days of storage at $22{ }^{\circ} \mathrm{C}$ (right). Note that the freshly prepared emulsion had finely distributed oil droplets. After 28 days, some bigger droplets and aggregates were visible, supporting the droplet size distribution data.

Generally, emulsions with higher GGM concentrations and lower oil concentrations were more stable during storage. The OOE, which had a GGM-to-oil ratio equal to or less than 0.25, (Fig. 4), underwent partial coalescence during storage, while OOE with GGM-to-oil ratio of around 0.5 , (Figs. 4 and 5a, b) were more stable. Thus, the critical GGM-to-oil ratio for our OOE was between 0.25 and 0.5 , lower than that obtained by our previous findings, which was between 1 and 2 [23]. Previously, we prepared emulsions using rapeseed oil and GGMs, which were precipitated using
Fig. 5 Optical microscopy images of freshly prepared $\mathrm{OOE}_{0.8 / 1.5}$ [emulsion containing $0.8 \%(\mathrm{w} / \mathrm{w}) \mathrm{GGM}$ and $1.5 \%(\mathrm{w} / \mathrm{w})$ oil] (left) and after 28 days of storage at $22{ }^{\circ} \mathrm{C}$ (right)
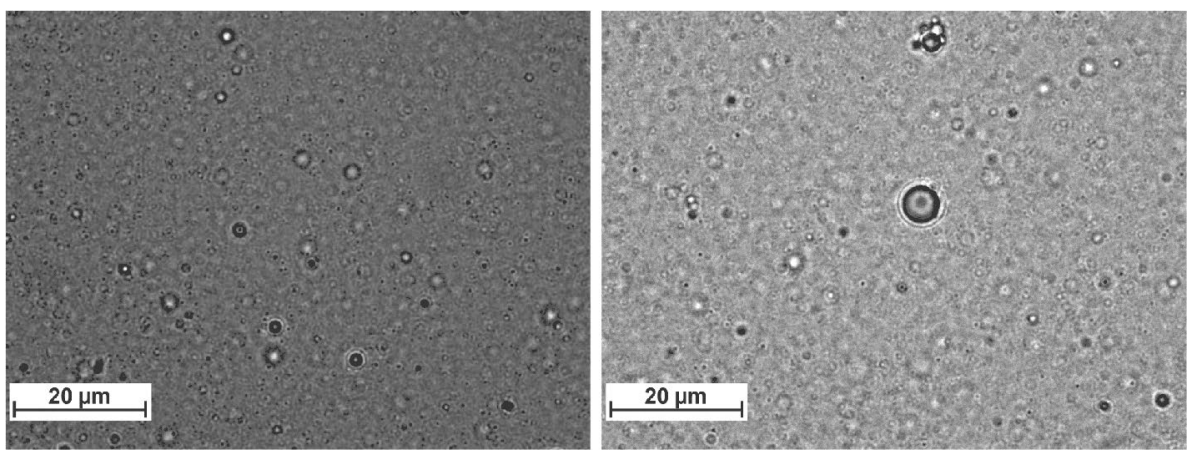
ethanol. The precipitation of GGMs by ethanol removes the ethanol-soluble, low molar mass GGM oligo/polysaccharides and free phenolic residues, which might help the physical and oxidative stabilization of the emulsion [20], and rapeseed oil has a different fatty acid composition than that in Table 1, which might have increased the critical GGMto-oil ratio as well. The lower the critical GGM-to-oil ratio, the higher the ability of the surfactant to physically stabilize an emulsion. Thus, using non-purified GGMs, more stable emulsions can be obtained compared to using ethanolprecipitated GGMs [20]. GGM might have stabilized the oil droplets by steric hindrance [22] thereby reducing the interaction between the oil droplets and other particles in the oat-based beverage.

The most stable emulsions $\left(\mathrm{OOE}_{0.8 / 1.5}, \mathrm{OOE}_{0.5 / 1}\right)$ were pasteurized at $80^{\circ} \mathrm{C}$ for $10 \mathrm{~min}$ to evaluate their stability against thermal treatments. Both samples showed a droplet size distribution (Fig. S2, Supporting Information) and a mean droplet diameter comparable with those of the nonpasteurized samples $(D[4,3]$ of $0.25 \pm 0.05$ and $D[3,2]$ of $0.05 \pm 0.01$ for both pasteurized and non-pasteurized samples), indicating excellent stability of the emulsions when subjected to the pasteurization treatment. Our findings are in agreement with the behavior highlighted by Chanamai and McClements [48] on gum Arabic and modified starchstabilized emulsions. Indeed, polysaccharides are not prone to unfolding or interface desorption induced by heat as in the case of protein-stabilized emulsions, so they confer thermal stability to polysaccharide-stabilized emulsions [12, 48].

\section{Oat-based beverages}

The OOE emulsions were used to produce oat-based beverages by adding the oat protein and the $\beta$-glucan suspensions. Figure 6a shows an example of the resulting beverage $\left(\mathrm{OBB}_{0.5 / 1}\right)$. The $\mathrm{OBB}$ beverages were brownish in color (Fig. 6a). The color of the OBB samples could have been due to OOE (in this case, the lignin compounds that are present in GGMs, as discussed previously) and $\beta$-glucan-rich flour, thus the color coming from GGM is not expected to affect the product's acceptability to consumers since the oat-based ingredients have a similar color. The samples appeared as suspensions of particles that tended to sediment over time (Fig. 6a, b). It is interesting to note that the stability of the oat-based beverages in terms of stability index (SI) seemed to be dependent on the GGM content rather the GGM-tooil ratio (Fig. 6b). The sedimentation was dependent on the emulsion formulation (Fig. $6 \mathrm{~b}$ ). $\mathrm{OBB}_{0.2 / 1.5}$ had the highest stability index (SI) over time, whereas, the sample based on the emulsion that contained the same amount of oil (1.5\%) but more GGMs $(0.8 \%)$, i.e., $\mathrm{OBB}_{0.8 / 1.5}$, had the lowest SI. The sedimentation could have been due to the presence of insoluble particles in the beverage and to the aggregation of oat proteins and oat $\beta$-glucan over time that increased the dimensions of the particles, which favored phase separation $[49,50]$. The presence of oat proteins and $\beta$-glucan led to the formation of aggregates that increased over time, forming a compact matrix that led to syneresis. However, the aggregates were dispersed easily by turning the container upside down, again forming a homogeneous dispersion similar to that observed in the freshly prepared samples.

The reduction of the SI also was reflected in the rheological properties of the OBB samples, with all OBB samples exhibiting a pseudoplastic behavior. Over time, the samples tended to shift towards a more liquid-like behavior (data not shown). This transition was accompanied by a marked reduction of the viscosity (Table 2 ), which possibly was due to the aggregation of the suspended particles which incorporated the oil droplets, followed by syneresis. Indeed, when a system undergoes aggregation, the rheological behavior of the resulting material can be altered and result in a reduction of the viscosity of the system itself [12]. However, oil did not separate from the beverage; rather, it remained dispersed as small oil droplets which can be re-dispersed by mixing the sample. No visible oil was detected on top of the sample, which
Fig. 6 Visual appearance of $\mathrm{OBB}_{0.5 / 1}(\mathbf{a})$ and stability indexes (SI) for oat-based beverages (b) after 1, 7, and 28 days of storage at $22{ }^{\circ} \mathrm{C}$
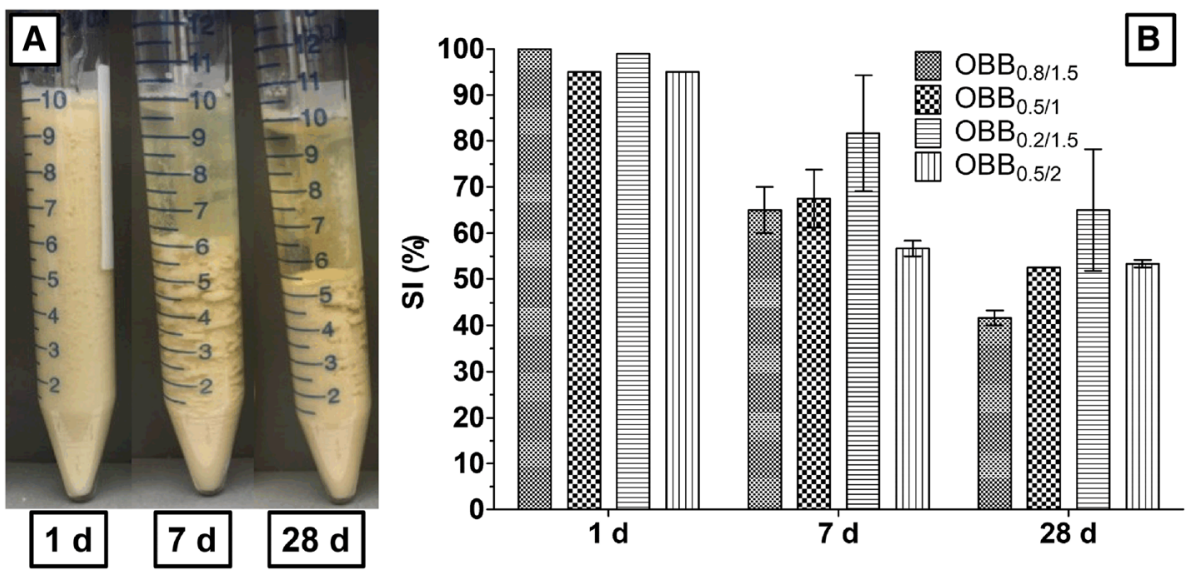
Table 2 Viscosity recorded at $100 \mathrm{~s}^{-1}$ for oat-based beverages $\left(\mathrm{OBB}_{0.8 / 1.5}, \mathrm{OBB}_{0.5 / 1}\right.$, $\mathrm{OBB}_{0.2 / 1.5}, \mathrm{OBB}_{0.5 / 2}$ ) after 1,7 , and 28 days of storage at $22{ }^{\circ} \mathrm{C}$

\begin{tabular}{lllr}
\hline Sample & \multicolumn{3}{c}{ Storage time (days) } \\
\cline { 2 - 4 } & 1 & \multicolumn{1}{c}{7} & \multicolumn{1}{c}{28} \\
\hline $\mathrm{OBB}_{0.8 / 1.5}$ & 78.1 & 23.3 & 9.2 \\
$\mathrm{OBB}_{0.5 / 1}$ & 62.9 & 19.1 & 6.2 \\
$\mathrm{OBB}_{0.2 / 1.5}$ & 79.7 & 28.5 & 11.4 \\
$\mathrm{OBB}_{0.5 / 2}$ & 34.1 & 13.8 & 7.0 \\
\hline
\end{tabular}

The standard error for all measurements was $\leq 0.1 \mathrm{mPa} \mathrm{s}$

indicated that the oil droplets in the oat-based beverage were stable over time. The stability of the oil droplets also was confirmed by the optical microscopy images. As an example, Fig. 7 shows the microstructure of freshly prepared $\mathrm{OBB}_{0.5 / 2}$ (left) and after 28 days of storage at $22{ }^{\circ} \mathrm{C}$ (right). Note that some of the oil droplets embedded in the heterogeneous matrix can be observed in both micrographs. These oil droplets were similar to those observed in the starting emulsion (Fig. S3, Supporting Information).

Finally, $\mathrm{OBB}_{0.2 / 1.5}$ was selected and pasteurized at $80{ }^{\circ} \mathrm{C}$ for $10 \mathrm{~min}$ to assess its stability against thermal treatments. Figure 8 shows the SI as a function of time for both pasteurized and non-pasteurized samples. Note that the pasteurized sample had a slower sedimentation velocity than the non-treated sample. (The slope of the first part of the curve was -0.0426 and $-0.2025 \% / \mathrm{h}$ for the pasteurized and non-pasteurized oat-based beverages, respectively). Also, the final value of SI in the pasteurized sample was greater than that of the non-treated sample, indicating a lower tendency for syneresis to occur. This could be linked to the microstructure of the sample, which was affected significantly by pasteurization (Fig. S4, Supporting Information). Indeed, a more homogeneous suspension with less aggregates was noticed when the pasteurized sample was compared with the non-treated sample (Fig. S4).

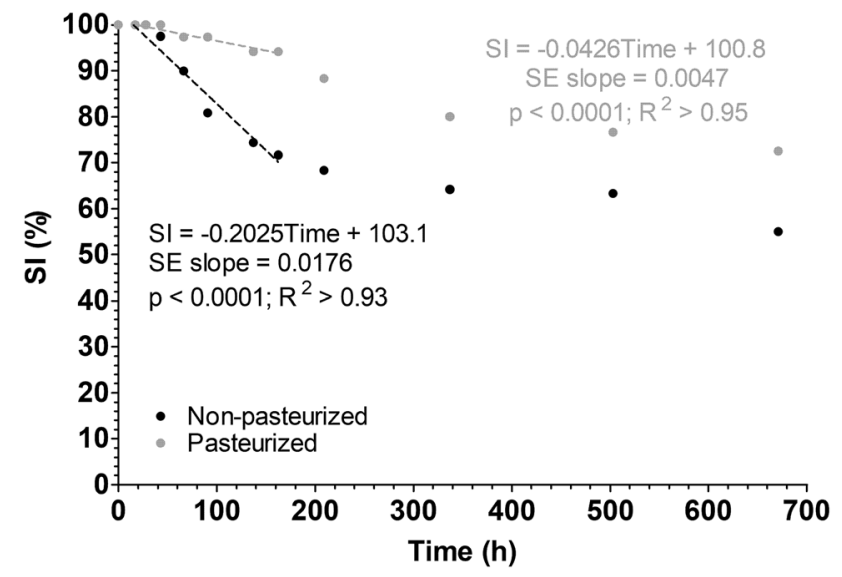

Fig. 8 Stability index (SI) as a function of storage time for pasteurized $\left(80{ }^{\circ} \mathrm{C}\right.$ for $\left.10 \mathrm{~min}\right)$ and non-pasteurized $\mathrm{OBB}_{0.2 / 1.5}$

\section{Conclusions}

GGM-stabilized emulsions are able to deliver oils rich in essential fatty acids in multi-component food matrices. The stability of the oil droplets embedded in the drinkable yogurt and oat-based beverages was maintained during storage at $4{ }^{\circ} \mathrm{C}$ and $22{ }^{\circ} \mathrm{C}$, respectively, over a prolonged period of time. Also, no oxidation of the cod liver oil in drinkable yogurt was observed during storage, and emulsions that contained oat oil had excellent thermal stability. Even though GGMs have no viscosity-enhancing properties like some other polysaccharides, and more studies are needed to better elucidate the interactions at molecular level between GGMs and other food components in complex matrices, it is evident that GGMs are versatile ingredients that can be used as a multi-purpose polysaccharide in the physical and chemical stabilization of emulsions. After appropriate safety evaluation, GGMs extracted from wood can be added as a new multi-functional hydrocolloid for food, cosmetic, pharmaceutical, and chemical applications. This study highlighted the great applicative potential of GGMs as novel,
Fig. 7 Optical microscopy images of freshly prepared $\mathrm{OBB}_{0.5 / 2}$ (left) and after 28 days of storage at $22{ }^{\circ} \mathrm{C}$ (right)
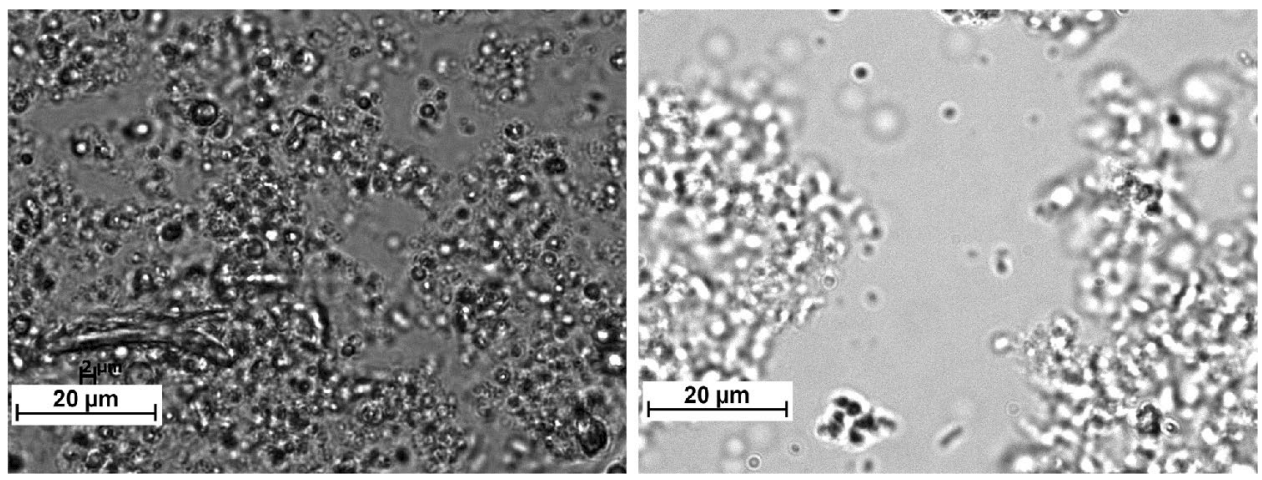
multi-purpose emulsifiers that can contribute to research related to the valorization of the side streams of the forest industry.

Acknowledgements Open access funding provided by University of Helsinki including Helsinki University Central Hospital. We thank Dr. Mari Lehtonen for performing analysis of the fatty acid composition and for assistance in the analysis of lipid oxidation, and Dr. AnnaMaija Lampi for fruitful discussion on lipid oxidation. We thank Oy Karl Fazer Ab for providing the oat beta-glucan and protein samples and Orkla Health for the cod oil sample without added antioxidants. We gratefully acknowledge The Academy of Finland (project number 305517) for providing the funding for this project, and we thank the members of the Project Advisory Board for fruitful discussions.

Funding This work was supported by the Academy of Finland (project number 305517).

\section{Compliance with ethical standards}

Conflict of interest The authors declare that they have no conflict of interest.

Compliance with ethics requirements This article does not contain any studies with animal or human subjects.

Open Access This article is distributed under the terms of the Creative Commons Attribution 4.0 International License (http://creativeco mmons.org/licenses/by/4.0/), which permits unrestricted use, distribution, and reproduction in any medium, provided you give appropriate credit to the original author(s) and the source, provide a link to the Creative Commons license, and indicate if changes were made.

\section{References}

1. Doyon M, Labrecque J (2008) Functional foods: a conceptual definition. Br Food J 110:1133-1149

2. Siró I, Kapolna E, Kapolna B, Lugasi A (2008) Functional food. Product development, marketing and consumer acceptance-a review. Appetite 51:456-467

3. Bigliardi B, Galati F (2013) Innovation trends in the food industry: the case of functional foods. Trends Food Sci Technol 31:118-129

4. Santeramo FG, Carlucci D, De Devitiis B, Seccia A, Stasi A, Viscecchia R, Nardone G (2018) Emerging trends in european food, diets and food industry. Food Res Int 104:39-47

5. McClements DJ, Decker EA, Park Y, Weiss J (2009) Structural design principles for delivery of bioactive components in nutraceuticals and functional foods. Crit Rev Food Sci Nutr 49:577-606

6. Al-Sheraji SH, Ismail A, Manap MY, Mustafa S, Yusof RM, Hassan FA (2013) Prebiotics as functional foods: a review. J Funct Foods 5:1542-1553

7. Tripathi MK, Giri SK (2014) Probiotic functional foods: survival of probiotics during processing and storage. J Funct Foods 9:225-241

8. Corbo MR, Bevilacqua A, Petruzzi L, Casanova FP, Sinigaglia M (2014) Functional beverages: the emerging side of functional foods commercial trends, research, and health implications. Compr Rev Food Sci Food Saf 13:1192-1206

9. Deora N, Deswal A (2018) Non-dairy based beverages: an insight. J Nutr Food Technol 1:1-4
10. McClements DJ (2018) Delivery by design (dbd): a standardized approach to the development of efficacious nanoparticle-and microparticle-based delivery systems. Compr Rev Food Sci Food Saf 17:200-219

11. Đorđević V, Balanč B, Belščak-Cvitanović A, Lević S, Trifković K, Kalušević A, Kostić I, Komes D, Bugarski B, Nedović V (2015) Trends in encapsulation technologies for delivery of food bioactive compounds. Food Eng Rev 7:452-490

12. McClements DJ (2016) Food emulsions: Principles, practices, and techniques. CRC Press, Boca Raton

13. Mikkonen KS, Xu C, Berton-Carabin C, Schroën K (2016) Spruce galactoglucomannans in rapeseed oil-in-water emulsions: efficient stabilization performance and structural partitioning. Food Hydrocoll 52:615-624

14. Daas PJ, Schols HA, de Jongh HH (2000) On the galactosyl distribution of commercial galactomannans. Carbohydr Res 329:609-619

15. Pitkänen L, Tuomainen P, Mikkonen KS, Tenkanen M (2011) The effect of galactose side units and mannan chain length on the macromolecular characteristics of galactomannans. Carbohydr Polym 86:1230-1235

16. Nishinari K, Williams PA, Phillips GO (1992) Review of the physico-chemical characteristics and properties of konjac mannan. Food Hydrocoll 6:199-222

17. Willför S, Sundberg K, Tenkanen M, Holmbom B (2008) Spruce-derived mannans-a potential raw material for hydrocolloids and novel advanced natural materials. Carbohydr Polym 72:197-210

18. Giummarella N, Lawoko M (2017) Structural insights on recalcitrance during hydrothermal hemicellulose extraction from wood. ACS Sustain Chem Eng 5:5156-5165

19. Nishimura H, Kamiya A, Nagata T, Katahira M, Watanabe $T$ (2018) Direct evidence for alpha ether linkage between lignin and carbohydrates in wood cell walls. Sci Rep 8:6538

20. Lehtonen M, Merinen M, Kilpeläinen PO, Xu C, Willför SM, Mikkonen KS (2018) Phenolic residues in spruce galactoglucomannans improve stabilization of oil-in-water emulsions. J Colloid Interface Sci 512:536-547

21. Kilpeläinen PO, Hautala SS, Byman OO, Tanner LJ, Korpinen RI, Lillandt MKJ, Pranovich AV, Kitunen VH, Willför SM, Ilvesniemi HS (2014) Pressurized hot water flow-through extraction system scale up from the laboratory to the pilot scale. Green Chem 16:3186-3194

22. Mikkonen KS, Merger D, Kilpeläinen P, Murtomaki L, Schmidt US, Wilhelm M (2016) Determination of physical emulsion stabilization mechanisms of wood hemicelluloses via rheological and interfacial characterization. Soft Matter 12:8690-8700

23. Bhattarai M, Pitkänen L, Kitunen V, Korpinen R, Ilvesniemi H, Kilpeläinen PO, Lehtonen M, Mikkonen KS (2018) Functionality of spruce galactoglucomannans in oil-in-water emulsions. Food Hydrocoll 86:154-161

24. Lehtonen M, Teräslahti S, Xu C, Yadav MP, Lampi A-M, Mikkonen KS (2016) Spruce galactoglucomannans inhibit lipid oxidation in rapeseed oil-in-water emulsions. Food Hydrocoll 58:255-266

25. Pitkänen L, Heinonen M, Mikkonen KS (2018) Safety considerations of phenolic-rich plant polysaccharides for food use: case study on softwood galactoglucomannan. Food Funct 9:1931-1943

26. Sibakov J, Abecassis J, Barron C, Poutanen K (2014) Electrostatic separation combined with ultra-fine grinding to produce $\beta$-glucan enriched ingredients from oat bran. Innov Food Sci Emerg Technol 26:445-455

27. Sibakov J, Myllymaki O, Holopainen U, Kaukovirta-Norja A, Hietaniemi V, Pihlava JM, Poutanen K, Lehtinen P (2011) Lipid removal enhances separation of oat grain cell wall material from starch and protein. J Cereal Sci 54:104-109 
28. Lehtinen P, Kaukovirta-Norja A, Sibakov J, Myllymäki O, Puotanen K, Philava J-M (2009) Functional oat ingredients-opportunities and challenges for food technology. Cereal Foods World $54: 267-271$

29. Yang C, Wang Y, Chen L (2017) Fabrication, characterization and controlled release properties of oat protein gels with percolating structure induced by cold gelation. Food Hydrocoll 62:21-34

30. Christie WW, Han X (2010) Lipid analysis. Woodhead Publishing Limited, Cambridge

31. Yang Z, Piironen V, Lampi AM (2017) Lipid-modifying enzymes in oat and faba bean. Food Res Int 100:335-343

32. Mirhosseini H, Tan CP, Hamid NSA, Yusof S (2008) Effect of arabic gum, xanthan gum and orange oil contents on $\zeta$-potential, conductivity, stability, size index and ph of orange beverage emulsion. Colloids Surf A 315:47-56

33. Henriksson G (2009) In: Gellerstedt G, Henriksson G (eds) Wood chemistry and wood biotechnology. De Gruyter, Berlin

34. Iiyama K, Nakano J, Migita N (1967) Studies on the color of lignin (ii). Japan Tappi J 21:157-164

35. Luo X, Zhou Y, Bai L, Liu F, Zhang R, Zhang Z, Zheng B, Deng Y, McClements DJ (2017) Production of highly concentrated oilin-water emulsions using dual-channel microfluidization: use of individual and mixed natural emulsifiers (saponin and lecithin). Food Res Int 96:103-112

36. Piorkowski DT, McClements DJ (2014) Beverage emulsions: recent developments in formulation, production, and applications. Food Hydrocoll 42:5-41

37. Spence C (2015) On the psychological impact of food colour. Flavour 4:21

38. Verbeke W (2005) Consumer acceptance of functional foods: socio-demographic, cognitive and attitudinal determinants. Food Qual Prefer 16:45-57

39. Lee WJ, Lucey JA (2010) Formation and physical properties of yogurt Asian-Aust. J Anim Sci 23:1127-1136

40. Bouzid H, Rabiller-Baudry M, Paugam L, Rousseau F, Derriche Z, Bettahar NE (2008) Impact of zeta potential and size of caseins as precursors of fouling deposit on limiting and critical fluxes in spiral ultrafiltration of modified skim milks. J Membr Sci 314:67-75
41. De Lorenzi L, Pricl S, Torriano G (1995) Rheological behaviour of low-fat and full-fat stirred yoghurt. Int Dairy J 5:661-671

42. Steele CM, James DF, Hori S, Polacco RC, Yee C (2014) Oral perceptual discrimination of viscosity differences for non-newtonian liquids in the nectar- and honey-thick ranges. Dysphagia 29:355-364

43. Vieira S, Zhang GD, Decker EA (2017) Biological implications of lipid oxidation products. J Am Oil Chem Soc 94:339-351

44. Sethi S, Tyagi SK, Anurag RK (2016) Plant-based milk alternatives an emerging segment of functional beverages: a review. J Food Sci Technol 53:3408-3423

45. Aaltonen K, Laurikka P, Huhtala H, Maki M, Kaukinen K, Kurppa $\mathrm{K}$ (2017) The long-term consumption of oats in celiac disease patients is safe: a large cross-sectional study. Nutrients 9:611

46. Lyly M, Ohls N, Lähteenmäki L, Salmenkallio-Marttila M, Liukkonen KH, Karhunen L, Poutanen K (2010) The effect of fibre amount, energy level and viscosity of beverages containing oat fibre supplement on perceived satiety. Food Nutr Res 54:2149

47. Biörklund M, van Rees A, Mensink RP, Önning G (2005) Changes in serum lipids and postprandial glucose and insulin concentrations after consumption of beverages with beta-glucans from oats or barley: a randomised dose-controlled trial. Eur J Clin Nutr 59:1272-1281

48. Chanamai R, McClements DJ (2002) Comparison of gum arabic, modified starch, and whey protein isolate as emulsifiers: influence of ph, $\mathrm{CaCl}_{2}$ and temperature. J Food Sci 67:120-125

49. Runyon JR, Nilsson L, Alftren J, Bergenstahl B (2013) Characterization of oat proteins and aggregates using asymmetric flow field-flow fractionation. Anal Bioanal Chem 405:6649-6655

50. Wu J, Zhao L, Li J, Jin S, Wu S (2017) Aggregation and gelation of oat beta-glucan in aqueous solution probed by NMR relaxometry. Carbohydr Polym 163:170-180

Publisher's Note Springer Nature remains neutral with regard to jurisdictional claims in published maps and institutional affiliations. 